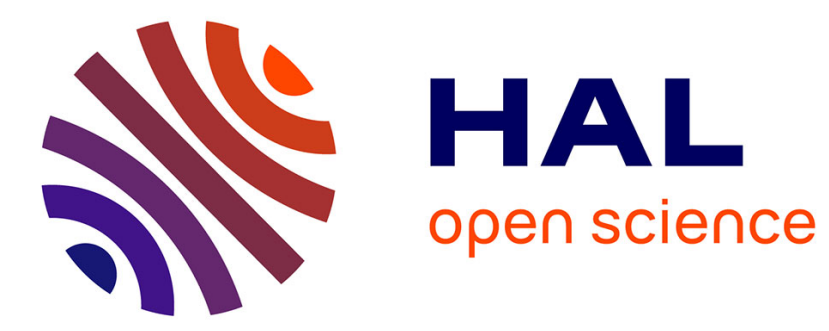

\title{
Sliding-mode control of retarded nonlinear systems via finite spectrum assignment approach
}

\author{
Toshiki Ogushi, Jean-Pierre Richard
}

\section{To cite this version:}

Toshiki Ogushi, Jean-Pierre Richard. Sliding-mode control of retarded nonlinear systems via finite spectrum assignment approach. IEEE Transactions on Automatic Control, 2006, 51 (9), pp.1527-1531. 10.1109/TAC.2006.880803 . inria-00131015

\section{HAL Id: inria-00131015 https://hal.inria.fr/inria-00131015}

Submitted on 14 Feb 2007

HAL is a multi-disciplinary open access archive for the deposit and dissemination of scientific research documents, whether they are published or not. The documents may come from teaching and research institutions in France or abroad, or from public or private research centers.
L'archive ouverte pluridisciplinaire HAL, est destinée au dépôt et à la diffusion de documents scientifiques de niveau recherche, publiés ou non, émanant des établissements d'enseignement et de recherche français ou étrangers, des laboratoires publics ou privés. 


\section{Sliding Mode Control of Retarded Nonlinear}

\section{Systems via Finite Spectrum Assignment}

\section{Approach}

Toshiki Oguchi, Member, IEEE, and Jean-Pierre Richard, Senior Member, IEEE

Toshiki Oguchi is with the Department of Mechanical Engineering, Tokyo Metropolitan University, 1-1, Minami-Osawa, Hachioji-shi, Tokyo 192-0397 Japan.

Jean-Pierre Richard is with LAGIS, CNRS UMR 8146, Ecole Centrale de Lille, 59651 Villeneuve d'Ascq Cedex, FRANCE

Corresponding author: Toshiki Oguchi e-mail: oguchi@ctrl.prec.metro-u.ac.jp 


\begin{abstract}
In the present study, a sliding mode control design method based on the finite spectrum assignment procedure is proposed. The finite spectrum assignment for retarded nonlinear systems can transform retarded nonlinear systems into delay-free linear systems via a variable transformation and a feedback, which contain the past values of the state. This method can be considered to be an extension of both the finite spectrum assignment for retarded linear systems with controllability over polynomial rings of the delay operator and the exact linearization for finite dimensional nonlinear systems. The proposed method is to design a sliding surface via the variable transformation used in the finite spectrum assignment and to derive a switching feedback law. The obtained surface contains not only the current values of the state variables but also the past values of the state variables in the original coordinates. The effectiveness of the proposed method is tested by an illustrative example.
\end{abstract}

\title{
Index Terms
}

time-delay systems, retarded nonlinear systems, finite spectrum assignment, exact linearization.

\section{INTRODUCTION}

Sliding Mode Control (SMC) [1] is a well-known robust control design approach. The fundamental idea of sliding mode control is to constrain the system trajectory on a predesigned hyperplane by a switching input. The greatest advantage of sliding mode control is its inherent insensitivity to uncertainties and disturbances which satisfy a certain structural condition called a matching condition. A standard sliding mode controller is designed in two steps. First, a sliding surface on which the system dynamics is governed is selected. Second, a switching control law to enforce the system trajectory on the selected surface is determined. A large number of SMC design methods [2], [3], [4], [5] for finite dimensional systems and a mathematical extension of differential inclusions to aftereffect systems [6] have been proposed, and several approaches have been developed for the SMC of linear time-delay systems [7], [8], [9], [10], [11], [12], [13], [14], [15], [16]. However, few approaches have been proposed for nonlinear systems with time-delay. A solution based on polytopic models was proposed in [17]. Bonnet et al. [18] considered linear, time-invariant and BIBO stable plants possessing a delay in the numerator. The output is measured via a relay sensor, and the result can be extended to saturated sensors. The result involves a "local inverse" of the sign operator. This means that this "inverse" has to be computed for a pre-defined reference signal, e.g. a sine function. The approach proposed herein is different from these methods. In the present paper, we consider a sliding mode control for retarded nonlinear systems, which are systems 
with time-delays in the state, and propose a new sliding mode controller design method based upon the finite spectrum assignment approach [19], [20] for retarded nonlinear systems. The finite spectrum assignment for retarded nonlinear systems can transform retarded nonlinear systems into delay-free linear systems via a variable transformation and a nonlinear feedback, which contain the previous values of the state. This method can be considered as an extension of both the finite spectrum assignment for retarded linear systems with controllability over polynomial rings and the exact linearization for finite dimensional nonlinear systems. The method proposed herein is to construct a sliding surface via a variable transformation introduced for the finite spectrum assignment. Since the variable transformation converts the retarded nonlinear system into a delay-free linear system, we can reduce a design problem of nonlinear manifolds in an infinite dimensional space to one of linear hyperplanes in a finite dimensional space. In addition, we derive a matching condition for the perturbations by means of the equivalent control approach. The obtained condition is a generalization for the time-delay systems of a well-known matching condition for finite dimensional systems.

\section{PReliminary}

At the beginning, we briefly introduce a finite spectrum assignment (FSA) method for retarded nonlinear systems [19], [20]. We consider the following retarded nonlinear systems with single input:

$$
\dot{\boldsymbol{x}}(t)=f\left(\boldsymbol{x}(t), \cdots, \boldsymbol{x}\left(t-\ell_{f_{k}}\right)\right)+g\left(\boldsymbol{x}(t), \cdots, \boldsymbol{x}\left(t-\ell_{g_{q}}\right)\right) u(t)
$$

where $\boldsymbol{x} \in \mathbb{R}^{n}, u \in \mathbb{R}$ and $f$ and $g$ are smooth vector fields with $g(0) \neq 0$. The initial condition is given by $\boldsymbol{x}(\theta)=\varphi(\theta)$ for $\theta \in\left[-\ell_{\max }, 0\right]$ where $\ell_{\max }$ denotes the maximum time-delay in the system (1). For the system, without loss of generality, we assume that the origin is an equilibrium point of system (1). By applying the pure delay operator $\sigma_{*}: \lambda(t) \mapsto \lambda\left(t-\ell_{*}\right)$ and $\sigma_{g_{0}}: \lambda(t) \mapsto \lambda(t)$, which is the identity map, system (1) can be rewritten as the following equation:

$$
\begin{aligned}
\dot{\boldsymbol{x}}(t) & =f\left(\boldsymbol{x}, \sigma_{f_{1}} \boldsymbol{x}, \cdots, \sigma_{f_{k}} \boldsymbol{x}\right)+\left\{g\left(\boldsymbol{x}, \cdots, \sigma_{g_{q}} \boldsymbol{x}\right) \sigma_{g_{0}}\right\} u(t) \\
& \triangleq \bar{f}(\boldsymbol{x}, \boldsymbol{\sigma})+\bar{g}(\boldsymbol{x}, \boldsymbol{\sigma}) u(t)
\end{aligned}
$$


Note that when the pure delay operator $\sigma_{i}$ is applied to $\bar{g}(\boldsymbol{x}, \boldsymbol{\sigma}) u(t)$, this operation means that

$$
\begin{aligned}
\sigma_{i} \bar{g}(\boldsymbol{x}, \boldsymbol{\sigma}) u(t) & =\sigma_{i}\left\{g\left(\boldsymbol{x}, \ldots, \sigma_{g_{q}} \boldsymbol{x}\right) \sigma_{g_{0}}\right\} u(t) \\
& =g\left(\sigma_{i} \boldsymbol{x}, \ldots, \sigma_{i} \sigma_{g_{q}} \boldsymbol{x}\right) \sigma_{i} u(t) \\
& =g\left(\boldsymbol{x}\left(t-\ell_{i}\right), \ldots, \boldsymbol{x}\left(t-\ell_{g_{q}}-\ell_{i}\right)\right) u\left(t-\ell_{i}\right) .
\end{aligned}
$$

Therefore $\sigma_{i} \bar{g}(\boldsymbol{x}, \boldsymbol{\sigma})=\left\{\sigma_{i} g\left(\boldsymbol{x}, \ldots, \sigma_{g_{q}}\right)\right\} \sigma_{i}$, where $\sigma_{g_{0}}$ can be omitted because $\sigma_{g_{0}}$ means the identity map.

For the system, we introduce the following definition.

\section{Definition 2.1: (delayed state derivative) [19]}

Let $\bar{f}(\boldsymbol{x}, \boldsymbol{\sigma}) \equiv f\left(\boldsymbol{x}(t), \boldsymbol{x}\left(t-\ell_{f_{1}}\right), \ldots, \boldsymbol{x}\left(t-\ell_{f_{k}}\right)\right)$ be an $n$-vector whose components are functions of $\boldsymbol{x}(t)$ and $\boldsymbol{x}\left(t-\ell_{f_{i}}\right), 1 \leq i \leq k, \bar{\phi}(\boldsymbol{x}, \boldsymbol{\sigma}) \equiv \phi\left(\boldsymbol{x}(t), \boldsymbol{x}\left(t-\ell_{\phi_{1}}\right), \ldots, \boldsymbol{x}\left(t-\ell_{\phi_{m}}\right)\right), 1 \leq i \leq m$, a real-valued function of $\boldsymbol{x}(t)$ and $\boldsymbol{x}\left(t-\ell_{\phi_{i}}\right), 1 \leq i \leq m$, and $\ell_{f_{i}}$ and $\ell_{\phi_{i}}$ positive time delays. Then, the derivative of $\bar{\phi}(\boldsymbol{x}, \boldsymbol{\sigma})$ along $\bar{f}(\boldsymbol{x}, \boldsymbol{\sigma})$ is defined by

$$
L_{\bar{f}} \bar{\phi}(\boldsymbol{x}, \boldsymbol{\sigma}) \stackrel{\text { def }}{=} \frac{\partial \bar{\phi}(\boldsymbol{x}, \boldsymbol{\sigma})}{\partial x(t)} \bar{f}+\sum_{i=1}^{m} \frac{\partial \bar{\phi}(\boldsymbol{x}, \boldsymbol{\sigma})}{\partial \sigma_{\phi_{i}} \boldsymbol{x}} \sigma_{\phi_{i}} \bar{f}(\boldsymbol{x}, \boldsymbol{\sigma})
$$

where $\sigma_{f_{i}}: \boldsymbol{x}(t) \mapsto \boldsymbol{x}\left(t-\ell_{f_{i}}\right)$ and $\sigma_{\phi_{i}}: \boldsymbol{x}(t) \mapsto \boldsymbol{x}\left(t-\ell_{\phi_{i}}\right)$ are the pure delay operators.

For $\gamma \geq 2$, the general form of higher-order derivatives can be denoted by $L_{\bar{f}}^{\gamma+1} \bar{\phi}(\boldsymbol{x}, \boldsymbol{\sigma})=L_{\bar{f}} L_{\bar{f}}^{\gamma} \bar{\phi}(\boldsymbol{x}, \boldsymbol{\sigma})$.

Throughout this paper, the delayed state derivative is used instead of the Lie derivative.

Now, for system (1), we assume that there exists a $C^{\infty}$-class real valued function $\bar{\phi}(\boldsymbol{x}, \boldsymbol{\sigma})$ of vector $\boldsymbol{x}(t)$ and the previous value of the vector $\boldsymbol{\sigma} \boldsymbol{x}$ which satisfy the following conditions for all $\boldsymbol{x}(t)$ and $\boldsymbol{\sigma} \boldsymbol{x}$ in a neighborhood $U$ of the origin:

1) $L_{\bar{g}} L_{\bar{f}}^{i} \bar{\phi}(\boldsymbol{x}, \boldsymbol{\sigma})=0$ for $i=0, \ldots, n-2$

2) $\frac{\partial L_{\bar{f}}^{n-1} \bar{\phi}(\boldsymbol{x}, \boldsymbol{\sigma})}{\partial \boldsymbol{x}(t)} \bar{g}(\boldsymbol{x}, \boldsymbol{\sigma}) \neq 0$

3) $\frac{\partial L_{\bar{f}}^{n-1} \bar{\phi}(\boldsymbol{x}, \boldsymbol{\sigma})}{\partial \sigma_{f_{j_{1}}} \cdots \sigma_{f_{j_{n-1}}} \boldsymbol{x}} \sigma_{f_{j_{1}}} \cdots \sigma_{f_{j_{n-1}}}(\bar{g}(\boldsymbol{x}, \boldsymbol{\sigma}))=0$ for $1 \leq j_{1} \leq k, 0 \leq j_{2}, \ldots, j_{n-1} \leq k$

where $\sigma_{f_{0}}: \boldsymbol{x}(t) \mapsto \boldsymbol{x}(t)$.

By applying a static feedback law defined for any $\boldsymbol{x}$ and $\boldsymbol{\sigma} \boldsymbol{x} \in U \subset \mathbb{R}^{n}$ :

$$
u(t)=\frac{-L_{\bar{f}}^{n} \bar{\phi}(\boldsymbol{x}, \boldsymbol{\sigma})+v(t)}{L_{\bar{g}} L_{\bar{f}}^{n-1} \bar{\phi}(\boldsymbol{x}, \boldsymbol{\sigma})} \triangleq \alpha(\boldsymbol{x}, \boldsymbol{\sigma})+\beta(\boldsymbol{x}, \boldsymbol{\sigma}) v(t)
$$


where $v(t)=\sum_{j=1}^{n} a_{j} z_{j}(t)$, and a variable transformation $\Phi: U \times \cdots \times U \rightarrow W \subset \mathbb{R}^{n}$ :

$$
\boldsymbol{z}(t)=\Phi(\boldsymbol{x}, \boldsymbol{\sigma}) \triangleq\left(\bar{\phi}(\boldsymbol{x}, \boldsymbol{\sigma}), \cdots, L_{\bar{f}}^{n-1} \bar{\phi}(\boldsymbol{x}, \boldsymbol{\sigma})\right)^{T}
$$

the system is transformed into

$$
\left\{\begin{array}{l}
\dot{z}_{i}(t)=z_{i+1}(t) \text { for } i=1, \ldots, n-1 \\
\dot{z}_{n}=-\sum_{i=1}^{n} a_{i} z_{i}(t)
\end{array}\right.
$$

Here, $a_{j}$ are chosen so that the closed loop system has $n$ poles in the preassigned position. We call this method a "finite spectrum assignment for retarded nonlinear systems by a static feedback". This method is an extension of the finite spectrum assignment [21], [22] for retarded linear systems which are controllable over the polynomial ring of $\sigma$. Moreover, it can be also recognized as an extension of the exact linearization of finite-dimensional nonlinear systems [23], [24]. Therefore, just as in the case of finite-dimensional systems, this controller design method can be also considered as a special case of the input-output linearization of retarded nonlinear systems [25] by replacing an output function $h(\boldsymbol{x})$ in [25] with a functional $\bar{\phi}(\boldsymbol{x}, \boldsymbol{\sigma})$ which leads to a relative degree $n$.

Remark 2.2: Note that even though the variable transformation (3) contains the delayed state, it is guaranteed that there exists a unique and causal inverse mapping $\boldsymbol{x}(t)=\Upsilon(\boldsymbol{z}, \boldsymbol{\sigma})$ from $(\boldsymbol{z}(t), \boldsymbol{\sigma} \boldsymbol{z})$ to $\boldsymbol{x}(t)$ in a neighborhood of the origin. The proof is given as the special case of Lemma 1 and 2 in Oguchi et al [25] by replacing $h(\boldsymbol{x})$ with $\bar{\phi}(\boldsymbol{x}, \boldsymbol{\sigma})$ and setting as $\rho=n$. This means that $\boldsymbol{x}=0$ holds exactly if $\boldsymbol{z}=0$ is maintained for some period. Moreover, this also means that the variable transformation (3) does not have any dynamics described by a difference equation. As a result, we can conclude that the local stability property of the system in $\boldsymbol{x}$-coordinates is the same as for the closed loop system in $z$-coordinates.

\section{Sliding Mode Control via FSA ApproACH}

\section{A. SMC design via FSA approach}

We consider the following nominal retarded nonlinear systems with single input as described by equation (2), and assume that system (2) can accomplish a finite spectrum assignment via static feedback in $U$. From 
this assumption, using a variable transformation $\boldsymbol{z}(t)=\Phi(\boldsymbol{x}, \boldsymbol{\sigma})$, system (2) can be transformed into

$$
\left\{\begin{array}{l}
\dot{z}_{i}(t)=z_{i+1}(t) \text { for } i=1, \ldots, n-1 \\
\dot{z}_{n}(t)=L_{\bar{f}}^{n} \bar{\phi}(\boldsymbol{x}, \boldsymbol{\sigma})+L_{\{\bar{g} u\}} L_{\bar{f}}^{n-1} \bar{\phi}(\boldsymbol{x}, \boldsymbol{\sigma}) .
\end{array}\right.
$$

Now, we design a switching surface such that $S(\boldsymbol{z})=0$, where $S(\boldsymbol{z})$ is defined as $S(\boldsymbol{z})=\sum_{i=1}^{n-1} s_{i} z_{i}+z_{n}$ and $s_{i}$ are coefficients of a monic Hurwitz polynomial of the form $p^{n}+s_{n-1} p^{n-1}+\cdots+s_{1}$. Note that this switching surface is described as a linear hyperplane but it consists of not only the current value of $\boldsymbol{x}$ but also the past values of $\boldsymbol{x}$. Therefore, this means that a design problem of infinite dimensional nonlinear manifolds can be reduced to a design problem of linear hyperplanes in a finite dimensional space by using a variable transformation (3).

Then the corresponding switching control law is defined as follows:

$$
\begin{cases}u(t)<\frac{-\sum_{i=1}^{n-1} s_{i} z_{i+1}-L_{\bar{f}}^{n} \bar{\phi}(\boldsymbol{x}, \boldsymbol{\sigma})}{L_{\bar{g}} L_{\bar{f}}^{n-1} \bar{\phi}(\boldsymbol{x}, \boldsymbol{\sigma})}-M & \text { if } \quad S(\boldsymbol{z}) \cdot L_{\bar{g}} L_{\bar{f}}^{n-1} \bar{\phi}(\boldsymbol{x}, \boldsymbol{\sigma})>0 \\ u(t)>\frac{-\sum_{i=1}^{n-1} s_{i} z_{i+1}-L_{\bar{f}}^{n} \bar{\phi}(\boldsymbol{x}, \boldsymbol{\sigma})}{L_{\bar{g}} L_{\bar{f}}^{n-1} \bar{\phi}(\boldsymbol{x}, \boldsymbol{\sigma})}+M & \text { if } \quad S(\boldsymbol{z}) \cdot L_{\bar{g}} L_{\bar{f}}^{n-1} \bar{\phi}(\boldsymbol{x}, \boldsymbol{\sigma}) \leq 0\end{cases}
$$

The following result shows that the system (2) generates a sliding motion by applying the above switching control law.

Theorem 3.1: The instantaneous state $\boldsymbol{z}(t)$ asymptotically moves toward the switching surface $S(\boldsymbol{z})=0$ and the system (2) generates a sliding motion if the switching control law satisfying (6) is applied to the system (2) on the operation region $U$.

Proof: Let $\mathcal{S}=\{\boldsymbol{z} \in W \mid S(\boldsymbol{z})=0\}$ and $\mathcal{S}^{c}=\{\boldsymbol{z} \in W \mid S(\boldsymbol{z}) \neq 0\}$. In addition, we select a positive definite function $V(\boldsymbol{z}, t)$ as $V(\boldsymbol{z}, t)=\frac{1}{2} S(\boldsymbol{z})^{2}$. Differentiation of $V(\boldsymbol{z}, t)$ with respect to time $t$ yields

$$
\begin{aligned}
\dot{V}(\boldsymbol{z}, t) & =S(\boldsymbol{z}) \frac{\partial S(\boldsymbol{z})}{\partial \boldsymbol{z}(t)} \frac{d \boldsymbol{z}}{d t} \\
& =S(\boldsymbol{z})\left(\sum_{i=1}^{n-1} s_{i} z_{i+1}+L_{\bar{f}}^{n} \bar{\phi}(\boldsymbol{x}, \boldsymbol{\sigma})+L_{\bar{g}} L_{\bar{f}}^{n-1} \bar{\phi}(\boldsymbol{x}, \boldsymbol{\sigma}) u(t)\right) .
\end{aligned}
$$

If $u(t)$ is chosen so that inequality (6) is satisfied, it is then easy to verify that $V(0, t)=\dot{V}(0, t)=0$ and $V(\boldsymbol{z}, t)=\dot{V}(\boldsymbol{z}, t)=0$ for all $\boldsymbol{z}(t) \in \mathcal{S}$. Since $L_{\bar{g}} L_{\bar{f}}^{n-1} \bar{\phi}(\boldsymbol{x}, \boldsymbol{\sigma})$ is continuous in $\boldsymbol{x}$ and $L_{\bar{g}} L_{\bar{f}}^{n-1} \bar{\phi}(\boldsymbol{x}, \boldsymbol{\sigma}) \neq 0$, there exists a $\delta>0$ such that $\left|L_{\bar{g}} L_{\bar{f}}^{n-1} \bar{\phi}(\boldsymbol{x}, \boldsymbol{\sigma})\right|>\delta$ for all $\boldsymbol{x}$ in a compact set $\mathcal{C}$ containing the origin, that is $s(\boldsymbol{x}, \boldsymbol{\sigma}) \equiv \sum_{i=1}^{n-1} s_{i} L_{\bar{f}}^{i-1} \bar{\phi}(\boldsymbol{x}, \boldsymbol{\sigma})+L_{\bar{f}}^{n-1} \bar{\phi}(\boldsymbol{x}, \boldsymbol{\sigma})=0$ in $\boldsymbol{x}$-coordinates. Therefore, the derivation of $V(\boldsymbol{z}, t)$ 
with respect to $t$ satisfies the following inequality.

$$
\dot{V}(\boldsymbol{z}, t)<-\sqrt{2} M\left|L_{\bar{g}} L_{\bar{f}}^{n-1} \bar{\phi}(\boldsymbol{x}, \boldsymbol{\sigma})\right| \sqrt{V}
$$

By solving this differential inequality, we obtain $2\left\{V^{\frac{1}{2}}(\boldsymbol{z}, t)-V^{\frac{1}{2}}\left(\boldsymbol{z}, t_{0}\right)\right\}<-\sqrt{2} \delta M\left(t-t_{0}\right)$. Setting $V\left(\boldsymbol{z}, t_{s}\right)=$ 0 at $t=t_{s}>t_{0}$ and $V\left(\boldsymbol{z}, t_{0}\right)=V_{0}$ at $t=t_{0}$, the inequality $t_{s}-t_{0}<\frac{\sqrt{2 V_{0}}}{\delta M}$ holds. As it is easily seen that the control law is asymptotically stabilizing the origin, any compact set $\mathcal{C}$ containing the origin is entered in a finite time. Thus $\left|L_{\bar{g}} L_{\bar{f}}^{n-1} \bar{\phi}(\boldsymbol{x}, \boldsymbol{\sigma})\right|>\delta$ and $V(\boldsymbol{z}, t)$ converges to zero in a finite time. Equivalently we can conclude that $\boldsymbol{z}(t)$ converges to $\mathcal{S}$ in a finite time or, in other words, that $S(\boldsymbol{z})$ converges to zero in a finite time. Therefore a sliding motion can be generated in a finite period.

From Theorem 3.1 we see that $S(\boldsymbol{z})$ converges to zero for all $\boldsymbol{z} \in \mathcal{S}^{c}$ and there exists a $t_{s}$ such that $S(\boldsymbol{z}(t))=0$ for all $t \geq t_{s}$, which is called a "reaching time". So, we consider the behavior of the system governed on the sliding hyperplane, i.e. $\boldsymbol{z} \in \mathcal{S}$. According to the above result, the equality $S(\boldsymbol{z}(t))=0$ holds in the sliding mode. The behavior of the reduced system on the hyperplane $S(\boldsymbol{z})=0$ is described by

$$
\left\{\begin{array}{l}
\dot{z}_{i}^{1}(t)=z_{i+1}^{1} \text { for } i=1, \ldots, n-2 \\
\dot{z}_{n-1}^{1}(t)=-\sum_{j=1}^{n-1} s_{j} z_{j}^{1}(t)
\end{array}\right.
$$

where $\boldsymbol{z}^{1}=\left(z_{1}, z_{2}, \cdots, z_{n-1}\right)^{T}$. If we choose $s_{j}$ for $j=1, \ldots, n-1$ as coefficients of a monic Hurwitz polynomial of the form $p^{n-1}+s_{n-1} p^{n-2}+\cdots+s_{1}$, we can show that $\lim _{t \rightarrow \infty} z^{1}(t)=0$, and this means that $\boldsymbol{z}(t)$ also converges to the origin in $z$-coordinates. As we mentioned in Remark 2.2, under condition 1), 2) and 3), the variable transformation $\boldsymbol{z}(t)=\Phi(\boldsymbol{x}, \boldsymbol{\sigma})$ is static and there exists a unique inverse mapping $\boldsymbol{x}(t)=\Upsilon(\boldsymbol{z}, \boldsymbol{\sigma})$ of the variable transformation in a neighborhood of the origin. Therefore, it is guaranteed that $\boldsymbol{x}(t)$ converges to the origin in $x$-coordinates. This means that the origin in $x$-coordinates is locally stabilized by applying the proposed sliding mode controller.

Remark 3.2: We emphasize that the above discussion gives the local nature of the stability of the equilibrium point $\boldsymbol{x}=0$. However if the conditions 1),2) and 3) are satisfied for any $\boldsymbol{x}$ and $\boldsymbol{\sigma} \boldsymbol{x} \in \mathbb{R}^{n}$ and the inverse mapping $\boldsymbol{x}(t)=\Upsilon(\boldsymbol{z}, \boldsymbol{\sigma})$ is globally defined, the global stability of the equilibrium point $\boldsymbol{x}=0$ is guaranteed. 


\section{B. Disturbance invariance property}

In this subsection, we focus on the behavior of the system on the sliding manifold and analyze the disturbance invariance property in the same way as Theorem 3 of Sira-Ramirez's paper [3] by replacing the conventional Lie derivative by the delayed state derivative. If the sliding mode controller is chosen so as to satisfy the inequality (6), sliding motion will occur at $t \geq t_{s}$, where $t_{s}$ is the reaching time. Hence, although the Sliding Mode Control system is discontinuous at the switching hyperplane $S(\boldsymbol{z})=0$, the behavior of the system is restricted to the switching hyperplane $S(\boldsymbol{z})=0$ for $t \geq t_{s}$. For such discontinuous systems of finite dimension, Filippov has proposed a system analysis method based on the equivalent control. In this section, using this analysis method, we consider the effect of uncertainties and disturbances in sliding mode.

If an ideal sliding motion is occurring, the system satisfies the following manifold invariant condition: $S(\boldsymbol{z})=$ 0 and $\frac{d S(\boldsymbol{z})}{d t}=0$. Since the switching surface $S(\boldsymbol{z})=0$ is rewritten as $s(\boldsymbol{x}, \boldsymbol{\sigma}) \equiv \sum_{i=1}^{n-1} s_{i} L_{\bar{f}}^{i-1} \bar{\phi}(\boldsymbol{x}, \boldsymbol{\sigma})+$ $L_{\bar{f}}^{n-1} \bar{\phi}(\boldsymbol{x}, \boldsymbol{\sigma})=0$ in $\boldsymbol{x}$-coordinates, the ideal sliding motions without system perturbations are described via the following conditions in $\boldsymbol{x}$ - coordinates:

$$
\left\{\begin{array}{l}
\sum_{i=1}^{n-1} s_{i} L_{\bar{f}}^{i-1} \bar{\phi}(\boldsymbol{x}, \boldsymbol{\sigma})+L_{\bar{f}}^{n-1} \bar{\phi}(\boldsymbol{x}, \boldsymbol{\sigma})=0 \\
L_{\bar{f}+\bar{g} u_{E Q}} s(\boldsymbol{x}, \boldsymbol{\sigma})=0
\end{array}\right.
$$

where $u_{E Q}(\boldsymbol{x}, \boldsymbol{\sigma})$ is a smooth feedback control law called the equivalent control. The second equation can be rewritten as

$$
\left(\frac{\partial s(\boldsymbol{x}, \boldsymbol{\sigma})}{\partial x} \bar{f}+\frac{\partial s(\boldsymbol{x}, \boldsymbol{\sigma})}{\partial \boldsymbol{\sigma} x} \boldsymbol{\sigma} \bar{f}\right)+\left(\frac{\partial s(\boldsymbol{x}, \boldsymbol{\sigma})}{\partial x} \bar{g} u_{E Q}+\frac{\partial s(\boldsymbol{x}, \boldsymbol{\sigma})}{\partial \boldsymbol{\sigma} x} \boldsymbol{\sigma}\left(\bar{g} u_{E Q}\right)\right)=0
$$

in which $\frac{\partial s(\boldsymbol{x}, \boldsymbol{\sigma})}{\partial \boldsymbol{\sigma} \boldsymbol{x}} \boldsymbol{\sigma}=\sum_{j=1}^{\gamma} \frac{\partial s(\boldsymbol{x}, \boldsymbol{\sigma})}{\partial \sigma_{j} \boldsymbol{x}} \sigma_{j}$ and $\sigma_{j}$ for $j=1, \ldots, \gamma$ are the entire delay operators contained in the switching function $s(\boldsymbol{x}, \boldsymbol{\sigma})$. Since system (2) satisfies condition 3, we obtain $\frac{\partial s(\boldsymbol{x}, \boldsymbol{\sigma})}{\partial \sigma_{j} x} \sigma_{j}\left(\bar{g}(\boldsymbol{x}, \boldsymbol{\sigma}) u_{E Q}\right)=0$ for $j=1, \ldots, \gamma$. Therefore, equation (9) is transformed into $\left(\frac{\partial s(\boldsymbol{x}, \boldsymbol{\sigma})}{\partial x} \bar{f}+\frac{\partial s(\boldsymbol{x}, \boldsymbol{\sigma})}{\partial \boldsymbol{\sigma} x} \boldsymbol{\sigma} \bar{f}\right)+\frac{\partial s(\boldsymbol{x}, \boldsymbol{\sigma})}{\partial x} \bar{g}(\boldsymbol{x}, \boldsymbol{\sigma}) u_{E Q}=0$. Under condition 2, the equivalent control is given explicitly as $u_{E Q}=-\left(\frac{\partial s(\boldsymbol{x}, \boldsymbol{\sigma})}{\partial x} \bar{g}\right)^{-1}\left(\frac{\partial s(\boldsymbol{x}, \boldsymbol{\sigma})}{\partial x}+\frac{\partial s(\boldsymbol{x}, \boldsymbol{\sigma})}{\partial \boldsymbol{\sigma} x} \boldsymbol{\sigma}\right) \bar{f}$. Substituting $u_{E Q}$ into equation (2), it follows that the motion of the system on the sliding manifold is governed by

$$
\dot{\boldsymbol{x}}=\left(I-\bar{g}\left(\frac{\partial s}{\partial x} \bar{g}\right)^{-1}\left(\frac{\partial s}{\partial x}+\frac{\partial s}{\partial \boldsymbol{\sigma} x} \boldsymbol{\sigma}\right)\right) \bar{f}(\boldsymbol{x}, \boldsymbol{\sigma})
$$


As a result, the behavior of the unperturbed system on the sliding mode is described by

$$
\left\{\begin{array}{l}
\dot{\boldsymbol{x}}=\left(I-\bar{g}\left(\frac{\partial s}{\partial x} \bar{g}\right)^{-1}\left(\frac{\partial s}{\partial x}+\frac{\partial s}{\partial \boldsymbol{\sigma} x} \boldsymbol{\sigma}\right)\right) \bar{f}(\boldsymbol{x}, \boldsymbol{\sigma}) \\
\sum_{i=1}^{n-1} s_{i} L_{\bar{f}}^{i-1} \bar{\phi}(\boldsymbol{x}, \boldsymbol{\sigma})+L_{\bar{f}}^{n-1} \bar{\phi}(\boldsymbol{x}, \boldsymbol{\sigma})=0
\end{array}\right.
$$

Next, consider a dynamical system with perturbation:

$$
\dot{\boldsymbol{x}}=\bar{f}(\boldsymbol{x}, \boldsymbol{\sigma})+\bar{g}(\boldsymbol{x}, \boldsymbol{\sigma}) u(t)+\bar{p}(\boldsymbol{x}, \boldsymbol{\sigma})
$$

where $\bar{p}(\boldsymbol{x}, \boldsymbol{\sigma})=p\left(\boldsymbol{x}(t), \boldsymbol{x}\left(t-\ell_{1}\right), \ldots, x\left(t-\ell_{k}\right)\right)$ represents uncertainties and/or disturbances. We obtain the following result for the relation between the structure of the perturbation and the influence of the perturbation upon the sliding behavior.

Theorem 3.3: The motion of system (10) on the sliding mode is independent of the perturbation $\bar{p}$ if and only if the perturbation vector $\bar{p}$ satisfies the matching condition

$$
\bar{p}(\boldsymbol{x}, \boldsymbol{\sigma}) \in \operatorname{span}\{\bar{g}(\boldsymbol{x}, \boldsymbol{\sigma})\} .
$$

Proof: This theorem is proved in a similar way as Theorem 3 in [3] owing to the introduction of the delayed state derivative. First, we show the necessity part. During the sliding mode, the manifold invariance condition $\frac{d s(\boldsymbol{x}, \boldsymbol{\sigma})}{d t}=0$ holds. Therefore, under the perturbed system, the equation $\left(\frac{\partial s(\boldsymbol{x}, \boldsymbol{\sigma})}{\partial \boldsymbol{x}}+\frac{\partial s(\boldsymbol{x}, \boldsymbol{\sigma})}{\partial \boldsymbol{\sigma} \boldsymbol{\sigma}}\right)(\bar{f}+$ $\left.\bar{g} u_{E Q}+\bar{p}\right)=0$ must be satisfied. Then, the equivalent control can be explicitly solved as follows: $u_{E Q}=$ $-\left(\frac{\partial s(\boldsymbol{x}, \boldsymbol{\sigma})}{\partial \boldsymbol{x}} \bar{g}\right)^{-1}\left(\frac{\partial s(\boldsymbol{x}, \boldsymbol{\sigma})}{\partial \boldsymbol{x}}+\frac{\partial s(\boldsymbol{x}, \boldsymbol{\sigma})}{\partial \boldsymbol{\sigma} \boldsymbol{x}} \boldsymbol{\sigma}\right)(\bar{f}+\bar{p})$. Substituting $u_{E Q}$ into equation (10), the ideal sliding behavior of the system is represented by $\dot{\boldsymbol{x}}=\left(I-\bar{g}\left(\frac{\partial s(\boldsymbol{x}, \boldsymbol{\sigma})}{\partial \boldsymbol{x}} \bar{g}\right)^{-1}\left(\frac{\partial s(\boldsymbol{x}, \boldsymbol{\sigma})}{\partial \boldsymbol{x}}+\frac{\partial s(\boldsymbol{x}, \boldsymbol{\sigma})}{\partial \boldsymbol{\sigma} \boldsymbol{\sigma}} \boldsymbol{\sigma}\right)\right)(\bar{f}+\bar{p})$. If the dynamics are independent of $\bar{p}$, then the following equation must hold: $\left(I-\bar{g}\left(\frac{\partial s(\boldsymbol{x}, \boldsymbol{\sigma})}{\partial \boldsymbol{x}} \bar{g}\right)^{-1}\left(\frac{\partial s(\boldsymbol{x}, \boldsymbol{\sigma})}{\partial \boldsymbol{x}}+\frac{\partial s(\boldsymbol{x}, \boldsymbol{\sigma})}{\partial \boldsymbol{\sigma} \boldsymbol{\sigma}}\right)\right) \bar{p}=0$. By solving this equation with respect to $\bar{p}$, we obtain $\bar{p}(\boldsymbol{x}, \boldsymbol{\sigma})=\bar{g} \cdot\left(\frac{\partial s(\boldsymbol{x}, \boldsymbol{\sigma})}{\partial \boldsymbol{x}} \bar{g}\right)^{-1}\left(\frac{\partial s(\boldsymbol{x}, \boldsymbol{\sigma})}{\partial \boldsymbol{x}}+\frac{\partial s(\boldsymbol{x}, \boldsymbol{\sigma})}{\partial \boldsymbol{\sigma} \boldsymbol{x}}\right) \bar{p}$, which implies that $\bar{p} \in \operatorname{span}\{\bar{g}(\boldsymbol{x}, \boldsymbol{\sigma})\}$.

Next, we show the sufficient condition. For any vector of the form $\bar{g}(\boldsymbol{x}, \boldsymbol{\sigma}) \eta(\boldsymbol{x}, \boldsymbol{\sigma})$, it follows that $\left(I-\bar{g}\left(\frac{\partial s(\boldsymbol{x}, \boldsymbol{\sigma})}{\partial \boldsymbol{x}} \bar{g}\right)^{-1}\left(\frac{\partial s(\boldsymbol{x}, \boldsymbol{\sigma})}{\partial \boldsymbol{x}}+\frac{\partial s(\boldsymbol{x}, \boldsymbol{\sigma})}{\partial \boldsymbol{\sigma} \boldsymbol{x}} \boldsymbol{\sigma}\right)\right) \bar{g} \eta=0$. Therefore, if $\bar{p} \in \operatorname{span}\{\bar{g}(\boldsymbol{x}, \boldsymbol{\sigma})\}$, the behavior of the system on the sliding manifold is obtained by $\dot{\boldsymbol{x}}=\left(I-\bar{g}\left(\frac{\partial s(\boldsymbol{x}, \boldsymbol{\sigma})}{\partial \boldsymbol{x}} \bar{g}\right)^{-1}\left(\frac{\partial s(\boldsymbol{x}, \boldsymbol{\sigma})}{\partial \boldsymbol{x}}+\frac{\partial s(\boldsymbol{x}, \boldsymbol{\sigma})}{\partial \boldsymbol{\sigma} \boldsymbol{\sigma}}\right)\right) \bar{f}$. This equation means that the influence of $\bar{p}$ on the equivalent dynamics is annihilated during sliding motion. Thus, the proof is complete. 
Theorem 3.3 is a generalization for time-delay systems of a well known matching condition for finite dimensional systems. From the above discussion, if a perturbation $\bar{p}$ is bounded and satisfies the matching condition (11), we can design a sliding mode controller so that system (10) is robust to the perturbation by choosing $M$ in switching control law (6) as $M>\left|\frac{L_{\bar{p}} s(\boldsymbol{x}, \boldsymbol{\sigma})}{L_{\bar{g}} L_{\bar{f}}^{-1} \bar{\phi}(\boldsymbol{x}, \boldsymbol{\sigma})}\right|=\left|\frac{L_{\bar{p}} L_{\bar{f}}^{n-1} \bar{\phi}(\boldsymbol{x}, \boldsymbol{\sigma})}{L_{\bar{g}} L_{\bar{f}}^{n-1} \bar{\phi}(\boldsymbol{x}, \boldsymbol{\sigma})}\right|$.

\section{ILLUSTRATIVE EXAMPLE}

We consider the following retarded nonlinear system:

$$
\dot{\boldsymbol{x}}(t)=\left(\begin{array}{c}
\left(1+\sigma x_{1}\right) x_{3}-x_{2} \\
x_{3}\left(\sigma x_{1}+\sigma x_{3}+\sigma^{2} x_{1} \cdot \sigma x_{3}-\sigma x_{2}\right) \\
x_{3}
\end{array}\right)+\left(\begin{array}{c}
0 \\
1+\sigma x_{1} \\
1
\end{array}\right) u(t)+\left(\begin{array}{c}
0 \\
\left(\cos \left(\sigma x_{3}\right)+1\right)\left(1+\sigma x_{1}\right) p(t) \\
\left(\cos \left(\sigma x_{3}\right)+1\right) p(t)
\end{array}\right) .
$$

where $\sigma \boldsymbol{x}(t) \triangleq \boldsymbol{x}(t-1)$ and $p(t)$ is any bounded disturbance. Then, the perturbation term $\bar{p} \triangleq\left(0,\left(\cos \left(\sigma x_{3}\right)+\right.\right.$ 1) $\left.\left(1+\sigma x_{1}\right) p(t),\left(\cos \left(\sigma x_{3}\right)+1\right) p(t)\right)^{T}$ satisfies the derived matching condition (11), and $\bar{\phi}(\boldsymbol{x})=x_{1}(t)$ satisfies conditions 1,2 and 3 for any $\boldsymbol{x}$ and $\boldsymbol{\sigma} \boldsymbol{x} \in \mathbb{R}^{n}$. Applying the variable transformation $\boldsymbol{z}(t):=\Phi(\boldsymbol{x}, \boldsymbol{\sigma})=$ $\left(x_{1}(t),\left(1+\sigma x_{1}(t)\right) x_{3}(t)-x_{2}(t), x_{3}(t)\right)^{T}$ system (12) is transformed into

$$
\dot{\boldsymbol{z}}(t)=\left[\begin{array}{ccc}
0 & 1 & 0 \\
0 & 0 & 1 \\
0 & 0 & 1
\end{array}\right] \boldsymbol{z}(t)+\left[\begin{array}{l}
0 \\
0 \\
1
\end{array}\right]\left(u(t)+\left(\cos \left(\sigma x_{3}\right)+1\right) p(t)\right)
$$

and $L_{\bar{g}} L_{\bar{f}}^{2} \bar{\phi}(\boldsymbol{x}, \boldsymbol{\sigma})=1$ holds. Choosing a sliding surface as $S(\boldsymbol{z})=s_{1} z_{1}+s_{2} z_{2}+z_{3}=0$, a switching control law that satisfies the following inequality is selected:

$$
\left\{\begin{array}{l}
u(t)<-s_{1} z_{2}-s_{2} z_{3}-x_{3}-M, \text { if } S(\boldsymbol{z})>0 \\
u(t)>-s_{1} z_{2}-s_{2} z_{3}-x_{3}+M, \text { if } S(\boldsymbol{z}) \leq 0
\end{array}\right.
$$

where $M$ is a positive constant satisfying $M>\frac{\left|L_{\bar{p}} L_{\bar{f}}^{2} \bar{\phi}(\boldsymbol{x}, \boldsymbol{\sigma})\right|}{\left|L_{\bar{g}} L_{\bar{f}}^{2} \phi(\boldsymbol{x}, \boldsymbol{\sigma})\right|}=\left|L_{\bar{p}} L_{\bar{f}}^{2} \bar{\phi}(\boldsymbol{x}, \boldsymbol{\sigma})\right|$. In this example, since the inverse transformation of $\boldsymbol{z}(t)=\Phi(\boldsymbol{x}, \boldsymbol{\sigma})$ is globally defined by $\boldsymbol{x}(t)=\left(z_{1}(t),-z_{2}(t)+\left(1+\sigma z_{1}\right) z_{3}(t), z_{3}(t)\right)^{T}$, the stability property of the equilibrium point $\boldsymbol{x}=0$ is the global character. Figures 1 and 2 show the behavior of the state variables $\boldsymbol{z}(t)$ and $\boldsymbol{x}(t)$, respectively, in a case of $s_{1}=1, s_{2}=2$ and $M=1$. The initial condition is given by $x_{1}(0)=-1.0, x_{2}(0)=2.0, x_{3}(0)=5.0$ and $\boldsymbol{x}(\theta)=0$ for $\theta<0$. This result shows that the sliding mode is invariant with respect to a perturbation satisfying the obtained matching condition. 


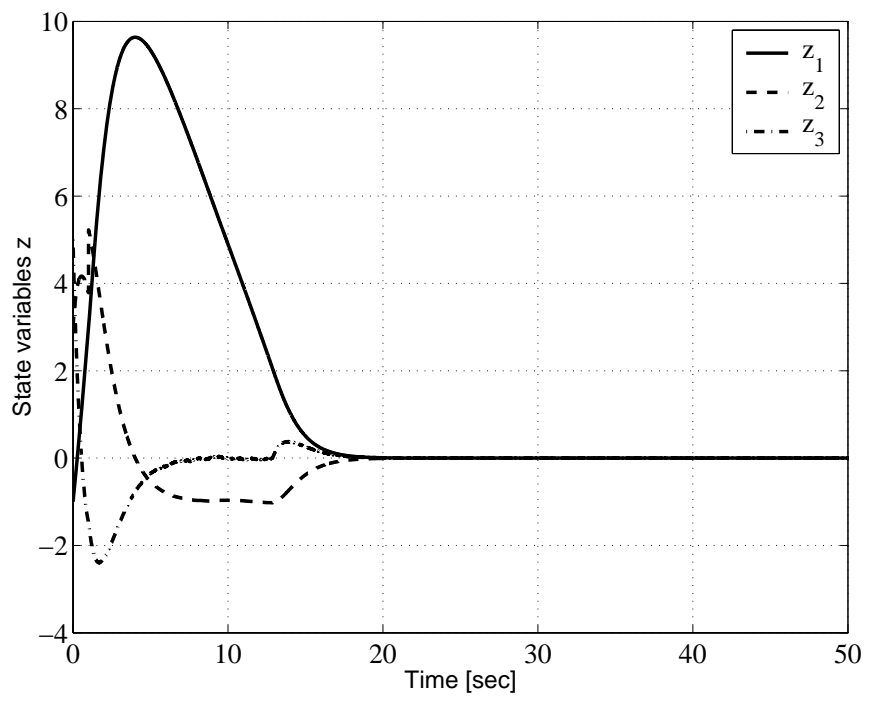

Fig. 1. Behavior of $z(t)$

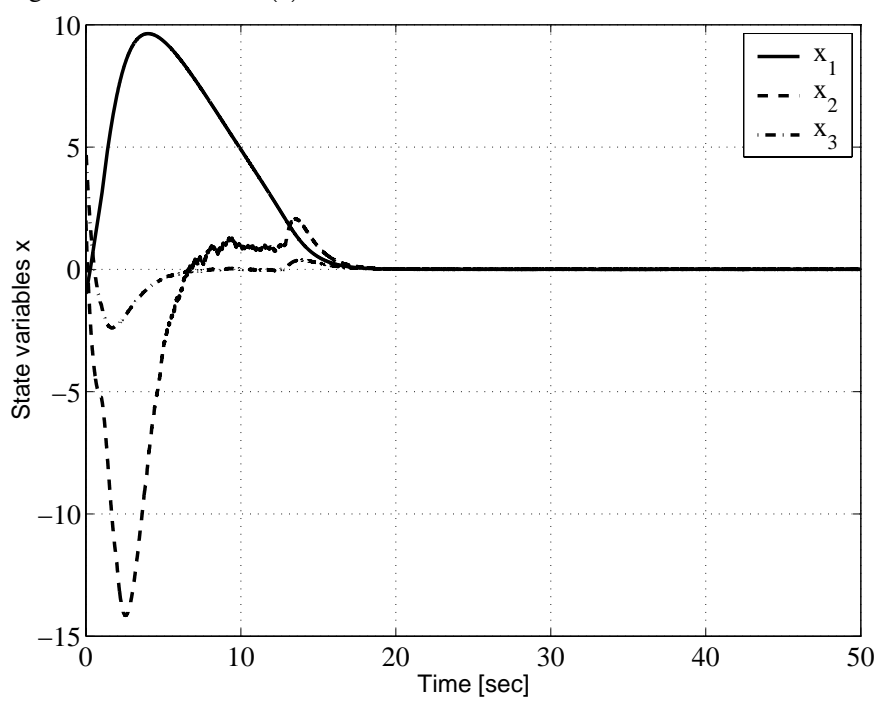

Fig. 2. Behavior of $x(t)$

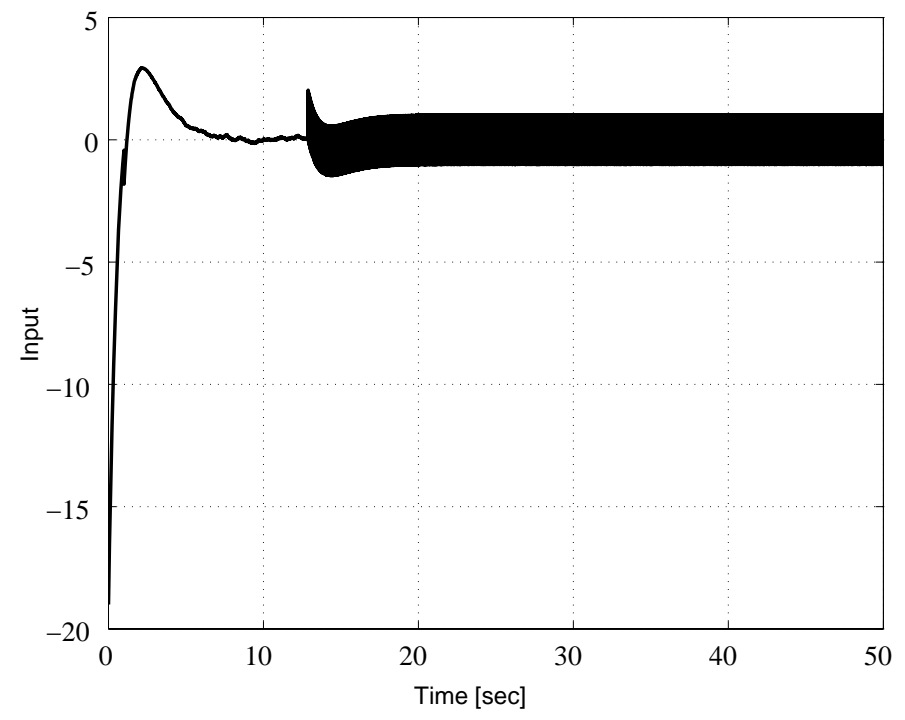

Fig. 3. Input $u(t)$ 


\section{CONClusion}

This paper considers a sliding mode control for retarded nonlinear systems. The proposed method involves the design of a sliding surface based on a variable transformation which contains the previous values of the state variables in the original coordinates and reduces the retarded nonlinear system to a delay-free linear system. By applying the variable transformation, a sliding mode controller can be designed based on the delay-free system. As a result, the retarded nonlinear system is asymptotically stabilized with the sliding mode controller based on the delay-free system. In addition, we derived a matching condition of perturbations by means of the equivalent control approach. The simulation result shows that the controller is robust to uncertainties and/or disturbances which satisfy the matching condition.

\section{ACKNOWLEDGMENT}

The authors thank the referees for their constructive suggestions which lead to an improvement of this manuscript.

\section{REFERENCES}

[1] V. Utkin, Sliding Modes in Control Optimization. Springer-Verlag, 1992.

[2] K. D. Young and Ü. Özgüner, Eds., Variable Structure Systems, Sliding Mode and Nonlinear Control, ser. $247 . \quad$ Springer, 1999.

[3] H. Sira-Ramirez, "Differential geometric methods in variable-structure control," Int. J. Control, vol. 48, no. 4, pp. 1359-1390, 1988.

[4] _ _ "Nonlinear variable systems in sliding mode: The general case," IEEE Trans. Automat. Contr., vol. 34, no. 11, pp. 1186-1188, 1989.

[5] W. Perruquetti and J. Barbot, Eds., Sliding Mode Control for Engineers, ser. Control Eng. Series. Marcel Dekker, 2002 , vol. 11.

[6] V. Kolmanovskii and A. Myshkis, Introduction to the theory and applications of functional differential equations. Kluwer Acad., Dordrecht, 1999.

[7] W. Aggoune, "Contribution à la stabilisation de systèmes non linéaires: Application aux systèmes non réguliers et aux systèmes à retards (in French)," INRIA Lorraine/CRAN, Univ. of Metz, France, June 1999.

[8] E. Cheres, S. Gutman, and Z. Palmor, "Stabilization of uncertain dynamic systems including state delay," IEEE Transactions on Automatic Control, vol. 34, no. 11, pp. 1199-1203, 1989.

[9] H. Choi, "An LMI approach to sliding mode control design for a class of uncertain time delay systems," in ECC99, European Control Conference, Karlsruhe, Germany, September 1999.

[10] S. Choi and J. Hedrick, "An observer-based controller design method for improving air/fuel characterization of spark ignition engines," IEEE Trans. Contr. Syst. Technol., vol. 6, no. 3, pp. 325-334, 1998.

[11] R. El-Khazaly, "Variable structure robust control of uncertain time-delay systems," Automatica, vol. 34, no. 3, pp. 327-332, 1998.

[12] F. Gouaisbaut, M. Dambrine, and J. Richard, "Robust control of delay systems: A sliding mode control design via LMIs," System and Control Letters, vol. 46, pp. 219-230, 2002. 
[13] X. Li and S. Yurkovich, Sliding Mode Control of Systems with Delayed State and Controls, K. Young and U. Ozguner, Eds. Springer Verlag, 1999, vol. 247.

[14] N. Luo and M. D. la Sen, "State feedback sliding mode controls of a class of time-delay systems," in ACC92 (American Control Conf.), Chicago, Illinois, USA, 1992, pp. 894-895.

[15] — , "State feedback sliding mode control of a class of uncertain time-delay systems," IEE proceedings-D, vol. 140, no. 4, pp. 261-274, 1993.

[16] K. Shyu and J. Yan, "Robust stability of uncertain time-delay systems and its stabilization by variable structure control," Int. J. of Control, vol. 57, pp. 237-246, 1993.

[17] F. Gouaisbaut, Y. Blanco, and J. Richard, "Robust control of nonlinear time delay systems: A sliding mode control design," Int. J. of Control, vol. 77, no. 2, 2004.

[18] C. Bonnet, J. Partington, and M. Sorine, "Robust control and tracking of a delay system with discontinuous non-linearity in the feedback," Int. J. Control, vol. 72, no. 15, pp. 1354-1364, 1999.

[19] T. Oguchi, A. Watanabe, and T. Nakamizo, "Finite spectrum assignment for nonlinear systems with non-commensurate delays," in Proceedings of the 14th IFAC World Congress, vol. C, Beijing, CHINA, July 1999, pp. 85-90.

[20] — - "Finite spectrum assignment for retarded nonlinear systems," Transactions of the Society of Instrument and Control Engineers, (in Japanese), vol. 36, no. 5, pp. 416-423, 2000.

[21] A. S. Morse, "Ring models for delay-differential systems," Automatica, vol. 12, pp. 529-531, 1976.

[22] E. D. Sontag, "Linear systems over commutative rings: A survey," Ricerche di Automatica, vol. 7, no. 1, pp. 1-34, 1976.

[23] A. Isidori, Nonlinear Control Systems - An Introduction, 3rd ed. Springer-Verlag, 1995.

[24] H. Nijmeijer and A. J. van der Schaft, Nonlinear Dynamical Control Systems, 1st ed. Springer-Verlag, 1990.

[25] T. Oguchi, A. Watanabe, and T. Nakamizo., "Input-output linearization of retarded non-linear systems by using an extension of Lie derivative," International Journal of Control, vol. 75, no. 8, pp. 582-590, 2002. 